\title{
Valorisation comparée \\ d'aliments à niveaux protéiques différents par des lapins sélectionnés sur la vitesse de croissance et par des lapins provenant d'élevages traditionnels. \\ II - Etude de la composition azotée et du métabolisme énergétique des muscles $L$. dorsi et $B$. femoris
}

\author{
J. OUHAYOUN et Danielle DELMAS
}

I.N.R.A., Laboratoire de Recherches sur l'Elevage du Lapin Centre de Recherches de Toulouse, F 31320 Castanet-Tolosan

\begin{abstract}
Résumé
La composition azotée et le métabolisme énergétique de deux muscles ( $L$. dorsi et $B$. femoris), prélevés sur des lapins âgés de 11 semaines, soit sélectionnés sur la vitesse de croissance, soit d'élevages traditionnels, sont étudiés. Les lapins sont nourris à volonté depuis le sevrage (4 semaines) avec des aliments isoénergétiques $(2420 \pm 70 \mathrm{kcal} \mathrm{ED} / \mathrm{kg})$ mais différant par la teneur en protéines $(17,2$ p. $100,13,8$ p. 100 ou 10,4 p. 100). La croissance musculaire est meilleure chez les lapins sélectionnés et, intra-type génétique, chez ceux qui consomment l'aliment le plus riche en protéines (tabl. 1). Les caractéristiques biochimiques différentielles des deux muscles sont déterminées par leur type métabolique : plus grande importance dans le $L$. dorsi des fractions azotées myofibrillaire, sarcoplasmique et non protéique et de l'activité enzymatique glycolytique (tabl. 2 et 4); supériorité du $B$. femoris en ce qui concerne la fraction du stroma et l'activité métabolique oxydative (tabl. 2 et 3). Le facteur génétique n'a d'effet que sur les fractions myofibrillaire (supériorité des lapins fermiers) et du stroma (supériorité des lapins sélectionnés) du $L$. dorsi. L'augmentation de la teneur en protéines de l'aliment a les mêmes effets sur les deux muscles des deux types génétiques : augmentation de la teneur en azote et accroissements corrélatifs de la fraction azotée sarcoplasmique et de l'activité enzymatique glycolytique.
\end{abstract}

\section{Introduction}

Le tissu musculaire constitue, avec le foie, une réserve labile de protéines dont les acides aminés sont mobilisables en cas de déficience du régime en matières azotées. Ces protéines peuvent être divisées en trois groupes principaux : les protéines myofibrillaires (myosine, actine, troponine et tropomyosine), les protéines sarcoplasmiques (enzymes de la glycolyse, créatine kinase, myoglobine...), les protéines du stroma (collagène, réticuline et élastine). Dans le tissu musculaire du lapin, les proportions de ces protéines évoluent rapidement après la naissance, mais à des rythmes différents selon les muscles; dès le $11^{\mathrm{e}}$ jour, la composition azotée relative du muscle adulte semble atteinte (VIGNERON, 1973). Cet équilibre présente des variations entre races (VIGNERON \& Nougues, 1974). Sa sensibilité aux niveaux en énergie et en protéines du régime 
alimentaire a été montrée chez le mouton (Asghar \& Yeates, 1979) et chez le poulet (ANTOSZEWSKA, 1979), mais pas, à notre connaissance, chez le lapin. La présente étude vise donc à déterminer l'équilibre des protéines musculaires chez des lapins de deux types génétiques qui, soumis à des équilibres alimentaires différents, se distinguent par le rendement de l'utilisation des matières azotées et la croissance musculaire (Ouhayoun \& Cheriet, 1983).

\section{Matériel et méthodes}

\section{A. Matériel animal}

L'expérimentation porte sur des lapereaux des deux sexes issus de la combinaison factorielle de deux types génétiques (souche I.N.R.A. 1027 , sélectionnée sur la vitesse de croissance; lapins d'élevages traditionnels) et de trois aliments isoénergétiques ( $2420 \pm 70 \mathrm{kcal}$ d'énergie digestible par $\mathrm{kg}$ ), différant par la teneur en protéines $(\mathrm{N} \times 6,25)(\mathrm{H}: 17,2$ p. $100 ; \mathrm{M}: 13,8$ p. 100 et $\mathrm{B}: 10,4$ p. 100). Les facteurs génétique et alimentaire et le mode d'échantillonnage des lapereaux au sevrage sont détaillés dans un mémoire séparé (OUHAYOun \& CHERIET, 1983). Les lapereaux utilisés dans la présente étude sont élevés en collectivité pendant 7 semaines après le sevrage (4 semaines), en six groupes définis par le plan factoriel. Ils sont alimentés à volonté. A l'âge de 11 semaines, quatre lapins sont tirés au hasard, au sein de chaque groupe et sacrifiés.

\section{B. Méthodes analytiques}

Les muscles Longissimus dorsi et Biceps femoris sont prélevés immédiatement après l'abattage et homogénéisés à la température de l'azote liquide dans un broyeur à billes Dangoumau.

Les protéines musculaires sont séparées par solubilité différentielle selon la méthode de Helander (1957). Les protéines sarcoplasmiques et myofibrillaires ainsi que l'azote non protéique sont extraits par une solution de KI 1,1 M dans le tampon phosphate $0,1 \mathrm{M}(\mathrm{pH} 7,4)$. Le résidu constitue le stroma. Les fractions myofibrillaires et sarcoplasmiques contenues dans le surnageant sont précipitées ensemble par l'acide trichloracétique et séparées, par centrifugation, de l'azote non protéique. Par ailleurs, les protéines sarcoplasmiques et l'azote non protéique sont extraits par un tampon phosphate $0,025 \mathrm{M}(\mathrm{pH} 7,4)$. La fraction sarcoplasmique est précipitée par l'acide trichloracétique et séparée par centrifugation.

Le métabolisme énergétique musculaire est estimé par mesure de l'activité d'une enzyme représentative de chacune des voies mitochondriale (isocitrate déshydrogénase, E.C. : 1-1-1-41) et sarcoplasmique (fructose-1,6-diphosphate aldolase, E.C. : 4-1-2-13). Les enzymes sont extraites selon la technique de ANSAY (1974) puis dosées en cinétique continue en suivant la vitesse d'oxydation de $\mathrm{NADH}+\mathrm{H}^{+}$(aldolase) et de réduction de NADP+ $(\mathrm{ICDH})$.

\section{Méthodes statistiques}

Les paramètres statistiques des données zootechniques et biochimiques sont calculés pour chacune des six cellules du schéma factoriel. L'effet du type génétique et du 
régime alimentaire sur les caractères pondéraux est estimé par analyse de variance selon un modèle croisé avec interaction. Un facteur supplémentaire : le muscle, est pris en considération pour analyser les données biochimiques.

\section{Résultats et discussion}

La vitesse de croissance moyenne des lapins sélectionnés (S) est supérieure à celle des lapins fermiers $(F)$. Les écarts relatifs entre types génétiques $\left(\frac{S-F}{F}\right.$ p. 100) :

18,3 p. $100 ; 30,2$ p. 100 et 26,8 p. 100 avec les aliments $H$, $M$ et $\mathbf{B}$, respectivement, sont nettement plus importants que ceux mesurés antérieurement chez des lapins élevés en cages individuelles (OuHayoun \& CHERIET, 1983). Intra-type génétique, les meilleurs gains moyens de poids vif pendant la période de contrôle sont obtenus avec les aliments les plus riches en protéines (tabl. 1).

Le développement des muscles $B$. femoris et $L$. dorsi est sensible, aussi, à l'effet des facteurs génétique et alimentaire, mais de façon différentielle. En effet, le muscle $B$. femoris représente une fraction assez constante du poids corporel dans les six cellules du plan factoriel $(0,54$ à 0,58 p. 100), malgré d'importants écarts entre les poids corporels moyens. Le muscle $L$. dorsi, par contre, est relativement moins développé chez les lapins légers. De développement plus tardif que le $B$. femoris (Vezrnhet et $a l ., 1972$ ), le $L$. dorsi subit donc une influence particulière des facteurs qui interviennent sur la vitesse de croissance postsevrage. L'existence d'une interaction hautement significative des facteurs «régime alimentaire» et «muscle» sur le poids du B. femoris et du $L$. dorsi (tabl. 2) résulte, en fait, de la dysharmonie de croissance de ces muscles pendant la période où les traitements alimentaires sont appliqués.

Intra-régime, les muscles $B$. femoris et $L$. dorsi ont la même teneur en azote total (tabl. 3 et 4), celle-ci ne varie pas, non plus, entre types génétiques (tabl. 2). Mais l'augmentation du niveau protéique du régime améliore la teneur en azote des deux muscles, tant chez les lapins sélectionnés que chez les fermiers. Cela confirme les résultats d'analyse portant sur des homogénats de carcasse (OuHAyoun \& CheRIET, 1983).

L'équilibre entre les différentes fractions azotées du B. femoris diffère considérablement de celui du $L$. dorsi (tabl. 2, 3 et 4). Les fractions myofibrillaire, sarcoplasmique et non protéique sont mieux représentées dans le $L$. dorsi. En revanche, la fraction du stroma est plus abondante dans le $B$. femoris. L'activité de l'isocitrate déshydrogénase, enzyme représentative du métabolisme oxydatif est plus forte dans le $B$. femoris que dans le $L$. dorsi, qui est riche en fibres $\alpha \mathrm{W}$, à contraction rapide et à métabolisme énergétique glycolytique (Vigneron, BaCou \& Ashmore, 1976). Intra-régime, seuls deux caractères du $L$. dorsi permettent de distinguer les lapins sélectionnés (S) des lapins fermiers (F) : les fractions protéiques myofibrillaire et du stroma. La première est moins importante chez les lapins sélectionnés; l'écart relatif entre les pourcentages

$\left(\frac{S-F}{F}\right.$ p. 100$)$ est compris entre $-2,2$ p. 100 et $-9,3$ p. 100 , selon les régimes 


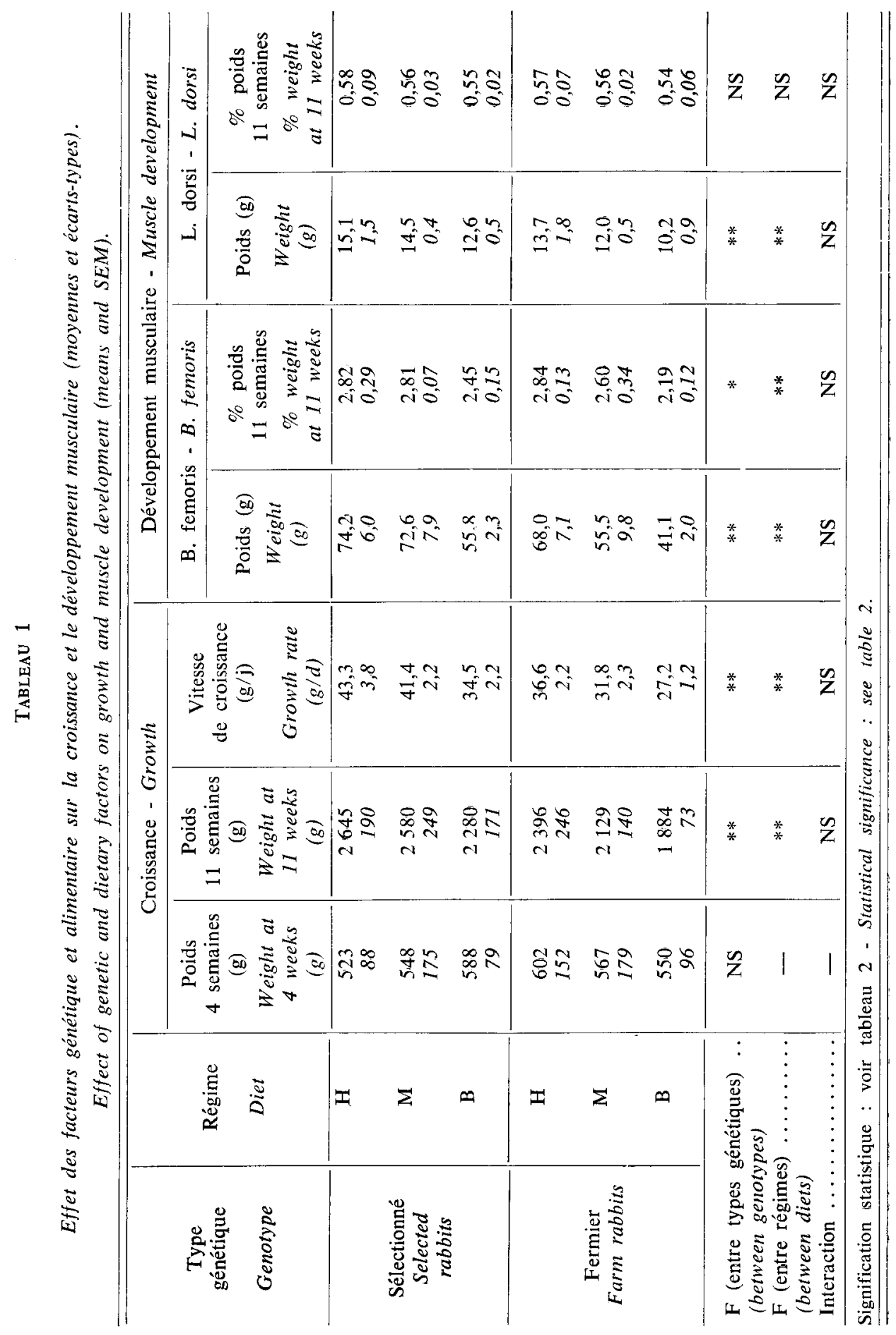


PROTÉINES MUSCULAIRES DU LAPIN

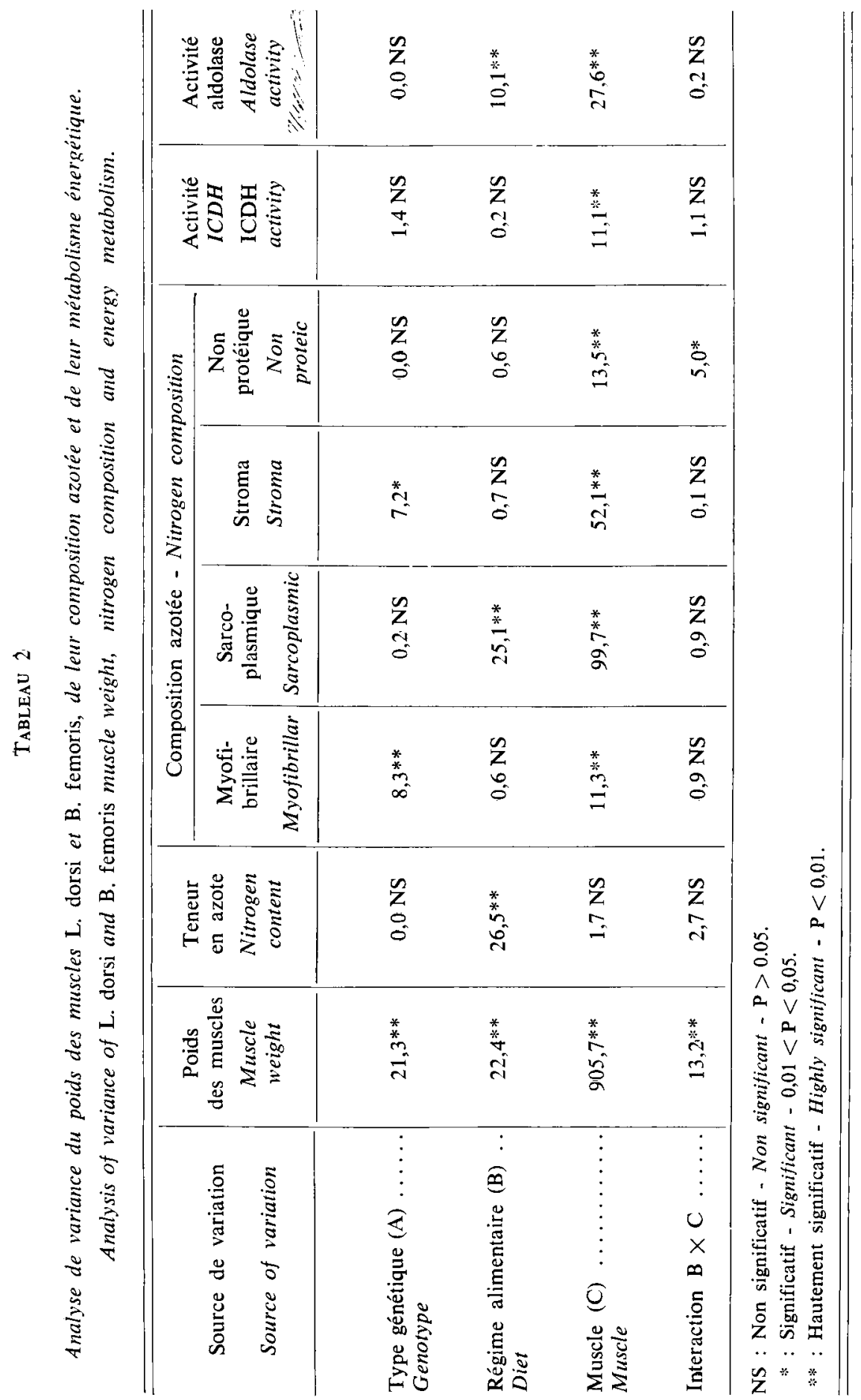




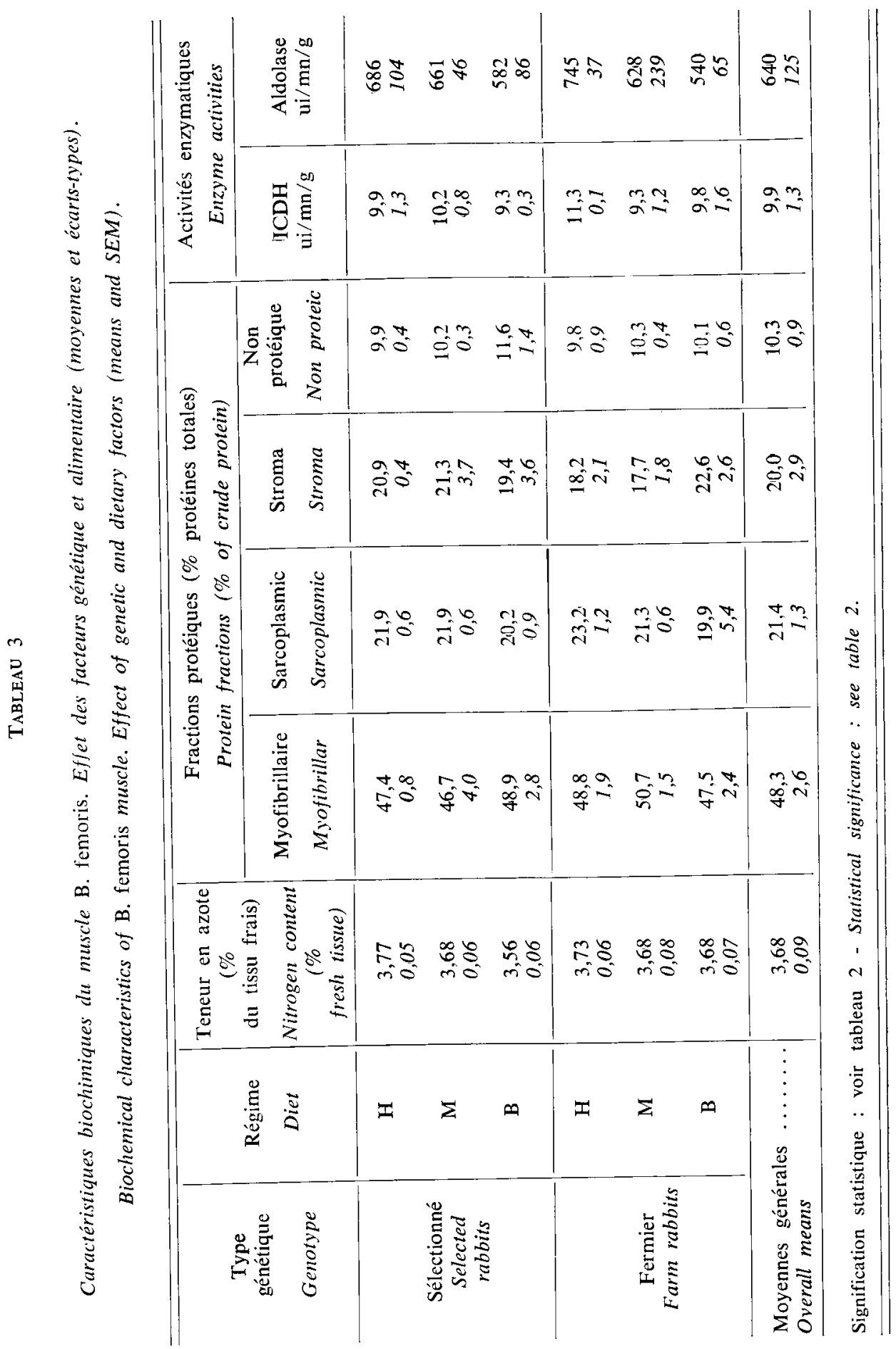




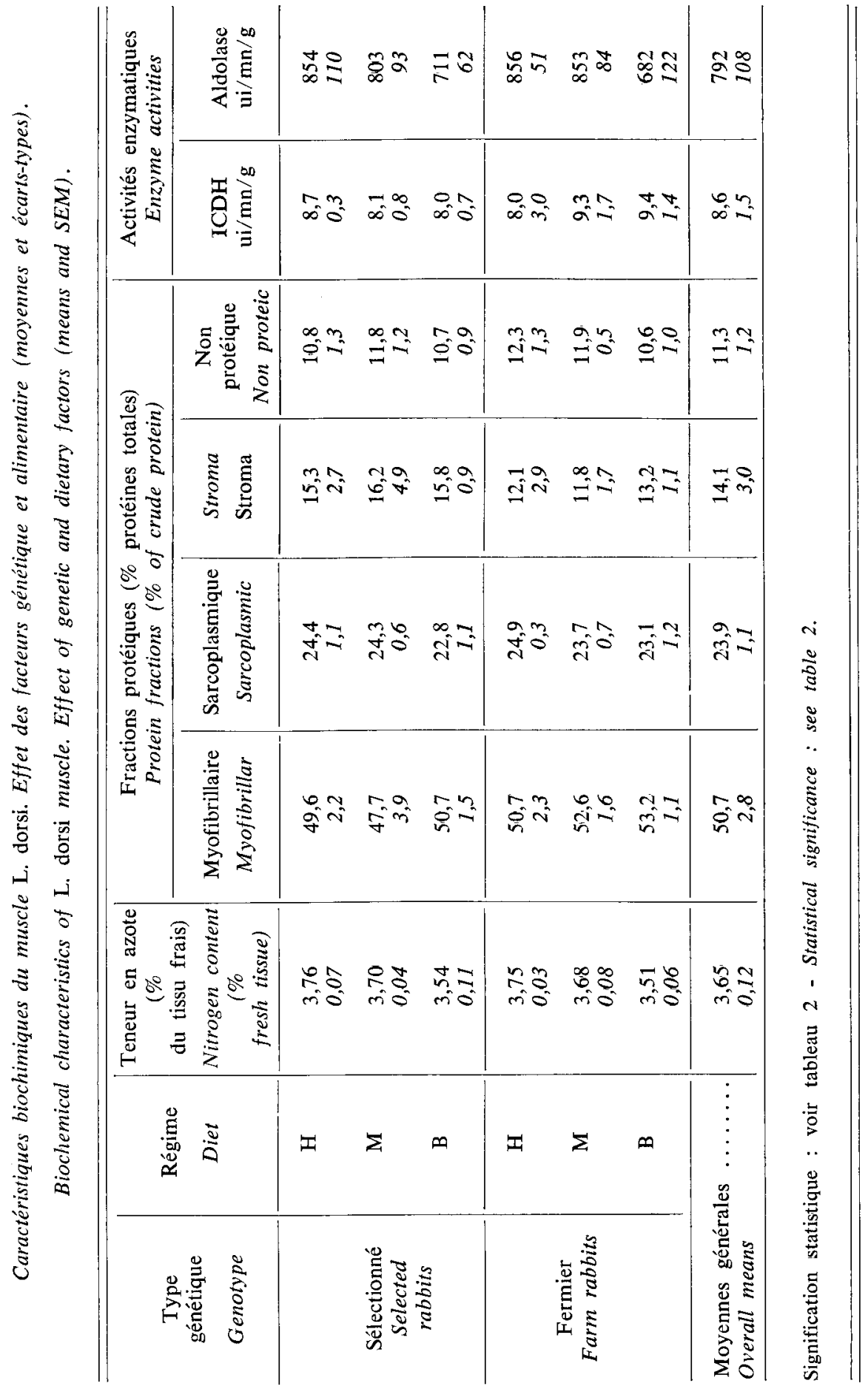


alimentaires. En revanche la fraction du stroma est plus développée : + 19,7 p. 100 $<\frac{\mathrm{S}-\mathrm{F}}{\mathrm{F}}<+37,3$ p. 100 .

Bien que les muscles $L$. dorsi et $B$. femoris se distinguent par leur précocité de développement pondéral et leurs caractéristiques métaboliques, ils réagissent de façon comparable aux traitements alimentaires. Une faible interaction entre les facteurs «régime» et «muscle» est toutefois à noter en ce qui concerne la fraction non protéique de lazote total.

La fraction sarcoplasmique est mieux représentée dans les muscles des lapins recevant les aliments les plus riches en protéines. ANTOSZEWSKa (1979), comparant la composition azotée de muscles de poulets nourris ad libitum ou rationnés, observe aussi une plus forte proportion de protéines sarcoplasmiques chez les animaux alimentés de façon intensive. Rosochackı (1979) remarque que les variations quantitatives des protéines sarcoplasmiques sont accompagnées de modifications de leur profil électrophorétique.

L'activité de la fructose-1,6-diphosphate aldolase, enzyme de la voie glycolytique est, aussi, plus élevée dans les muscles des lapins à croissance rapide.

Les variations corrélatives de l'activité aldolasique et de la teneur en protéines sarcoplasmiques sont attribuables au fait que, selon SCOPEs (1970), 70 p. 100 de ces dernières sont impliquées dans le processus glycolytique.

Ashmore, Tomprins \& Doerr (1972) ont montré que les fibres musculaires de type $\alpha \mathrm{W}$ dérivent des fibres $\alpha \mathbf{R}$ au cours de la croissance. Cette transition se traduit, au plan biochimique, par une augmentation de l'activité du métabolisme énergétique glycolytique et par une réduction de lactivité enzymatique oxydative dans le tissu musculaire (Bacou \& Vigneron, 1976 ; Ouhayoun, Delmas \& Poujardieu, 1982). Or, il apparaît, dans le présent travail, que chez les lapins dont la croissance est favorisée par le niveau élevé de l'apport protéique alimentaire, les muscles manifestent une activité glycolytique et une teneur en protéines sarcoplasmiques élevées. Si cela était la traduction d'un équilibre entre fibres musculaires favorable au type $\alpha \mathrm{W}$, l'activité enzymatique oxydative devrait corrélativement, être plus faible; mais il n'en est rien.

Cet aspect mérite d'être approfondi, en particulier en prenant en considération le facteur «sexe» qui, d'après Vigneron (1973) et Vigneron \& Nougues (1974), aurait une certaine influence sur l'équilibre des fractions azotées musculaires.

Ces premiers résultats montrent que l'amélioration de la vitesse de croissance du lapin, obtenue par augmentation de la teneur en protéines de l'aliment, peut conduire à une représentation accrue des fibres $\alpha \mathrm{W}$ dans le tissu musculaire ou, au moins, à une augmentation de l'activité de leur équipement enzymatique glycolytique. Cet effet est comparable à celui qui est attendu de la sélection sur la vitesse de croissance, compte tenu, d'une part des valeurs d'héritabilité du pH ultime des muscles et de leur teneur en myoglobine et, d’autre part, de l’antagonisme génétique existant entre ces caractères et les performances productives (Ouhayoun, Rouvier \& Poujardieu, 1974 ; Ouhayoun et al., 1975 ; Ouhayoun, Delmas \& Poujardieu, 1982). 


\section{Summary}

Comparative utilization of diets with different crude protein levels in rabbits selected on growth rate and in farm rabbits

\section{2 - Nitrogenous composition and energy metabolism of L. dorsi and $\mathbf{B}$. femoris muscles}

Nitrogenous composition and energy metabolism of two muscles (L. dorsi and B. femoris) sampled in 11-week old rabbits either selected on growth rate or issued from conventional raising units, were studied. Rabbits were fed ad libitum from weaning (4 weeks) isoenergetic diets $(2420 \pm 70 \mathrm{kcal} \mathrm{DE} / \mathrm{kg})$ with different crude protein levels $(17.2$ p. $100,13.8$ p. 100 or 10.4 p. 100). Muscle growth was higher in selected rabbits and within genotype, in rabbits ingesting the diet with the highest protein level (tabl. 1). The differential biochemical characteristics of both muscles depended on their metabolic type : in $L$. dorsi the myofibrillar, sarcoplasmic and non protein nitrogenous fractions as well as the glycolytic enzyme activity were larger (tabl. 2 and 4 ); in the $B$. femoris, the stroma fraction was larger and the oxydative metabolic activity was higher (tabl. 2 and 3). The genetic factor only affected the myofibrillar (superiority of farm rabbits) and the stroma fractions (superiority of selected rabbits) of $L$. dorsi. The increase in the protein content of the diet had the same effects on both muscles of both genotypes : increase in the nitrogen content and correlative increase of both the sarcoplasmic nitrogenous fraction and the glycolytic enzyme activity.

\section{Références bibliographiques}

ANSAY M., 1974. Développement biochimique du muscle de foetus bovin. 3 - Enzymes et différenciation. Ann. Biol. anim. Bioch. Biophys., 14, 105-116.

ANTOSZEwSKA B., 1979. Effect of feeding level on the content of basic protein fractions in the pectoral and leg muscles of growing White Plymouth Rock hens. Pr. Mater. Zootech., 18, 77-91.

Asghar A., Yeates N.T.M., 1979. Muscle characteristics and meat quality of lambs grown on different nutritional planes. 2 - Chemical and biochemical effects on muscle. Agric. Biol. Chem., 43, 437-444.

Ashmore C.R., Tompkins G., Doerr L., 1972. Postnatal development of muscle fibre types in domestic animals. J. Anim. Sci., 34, 37-41.

Bacou F., Vigneron P., 1976. Evolution périnatale des voies métaboliques glycolytique et oxydative de divers types de muscles squelettiques du lapin et du poulet. $A n n$. Biol. anim. Bioch. Biophys., 16, 675-686.

Helander E., 1957. On quantitative muscle protein determination. Acta physiol. Scand., Suppl., 141, 9-95.

Ouhayoun J., Rouvier R., Poujardieu B., 1974. Relations génétiques entre les performances de croissance pondérale et le métabolisme du tissu musculaire de lapin.

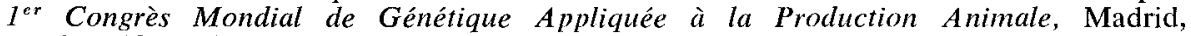
octobre 1974, 521-528.

Ouhayoun J., Rouvier R., Valin C., Lacourt A., 1975. Variation génétique de l'évolution post mortem du pH du tissu musculaire du lapin. Ann. Zootech., 24, 148 (Abst.).

Ouhayoun J., Delmas Danielle, Poujardieu B., 1982. Variabilität der Myoglobinkonzentration im Kaninchenmuskel-Beziehungen zum Energiestoffwechsel. II Internationales Kolloquium, Das Kaninchen als Modeltier und Züchtungsobjekt, Wilhelm Pieck Universität, Rostock, September 1982, Teil 1, 10 pp.

Ouhayoun J., Cheriet S., 1983. Valorisation comparée d'aliments à niveaux protéiques différents par les lapins sélectionnés sur la vitesse de croissance et par des lapins 
provenant d'élevages traditionnels. 1 - Etude des performances productives et de la composition du gain de poids. Ann. Zootech., 32, 257-276.

Rosochacki S.J., 1979. Effect of nutrition level on the composition of sarcoplasmic proteins in skeletal muscles of chicken. Pr. Mater. Zootech., 19, 71-85.

SCOPES L.K., 1970. Characterisation and study of sarcoplasmic proteins. In : The Physiology and Biochemistry of Muscle as a Food, vol. II, Univ. Wisc. Press., 471-492.

Vezinhet A., Rouvier R., Dulor J.P., Cantier J., 1972. Allométrie de croissance chez le lapin. 3 - Principales régions du système musculaire. Ann. Biol. anim. Bioch. Biophys., 12, 33-45.

Vigneron P., 1973. Evolution de la composition azotée des muscles psoas major et longissimus dorsi, de la naissance à 3 semaines chez le lapin. Ann. Biol. anim. Bioch. Biophys., 13, 553-563.

Vigneron P., Nougues J., 1974. Etude de la variation de la composition azotée du muscle psoas major chez trois races de lapins et leurs croisements réciproques. A $\mathrm{m}$. Biol. anim. Bioch. Biophys., 14, 131-143.

Vigneron P., Bacou F., Ashmore C.R., 1976. Distribution heterogeneity of muscle fiber types in the rabbit longissimus muscle. J. Anim. Sci., 43, 985-988. 